\title{
Phase Separation Process in Polymer Systems III. Spinodal Decomposition in the Critical Mixture of Polystyrene and Poly(methylphenylsiloxane) and Scaling Analysis
}

\author{
Shuichi NoJima, Yutaka OHYAMA, \\ Masashi YAMAGUCHI, and Takuhei NoSE \\ Department of Polymer Chemistry, Tokyo Institute of Technology, \\ Ookayama, Meguro-ku, Tokyo 152, Japan
}

(Received July 16, 1982)

\begin{abstract}
Spinodal decomposition in a critical mixture of polystyrene and poly(methylphenylsiloxane) was studied by light scattering. The maximum intensity of scattered light, $I_{\mathrm{m}}$, and the corresponding wave number, $k_{\mathrm{m}}$, varied with the phase separation time $t$ according to the following power-law relationships: $I_{\mathrm{m}} \propto t^{\theta}$ and $k_{\mathrm{m}} \propto t^{\phi}$ with $\theta=2.66( \pm 0.43)$ and $\phi=-0.90$ $( \pm 0.20)$. These values of $\theta$ and $\phi$ were three times as large as those previously obtained for the offcritical mixtures. $k_{\mathrm{m}}$ and $t$ were reduced to scaled values by the correlation length and diffusion coefficient evaluated from static and dynamic light-scattering measurements in the homogeneous region. The plots of the scaled $k_{\mathrm{m}}$ against the scaled $t$ gave two composite curves corresponding to the critical and off-critical mixtures and were compared with typical theoretical predictions and experimental results for binary mixtures of small molecules and a polymer solution.

KEY WORDS Spinodal Decomposition / Polymer Blend / Phase Separation / Light Scattering /
\end{abstract}

In the first paper of this series, ${ }^{1}$ we reported the angular dependence of scattered light (the spinodal ring) at the early stage of phase separation for polystyrene-poly(methylphenylsiloxane) mixtures as a function of phase separation time $t$, concentration, and quench depth. It was found that the maximum intensity $I_{\mathrm{m}}$ and the corresponding wave number $k_{\mathrm{m}}$ of the spinodal ring changed with $t$, following power-laws with the same exponents for any concentration and quench depth studied. For a better understanding of the temperature- and concentration-dependence of $I_{\mathrm{m}}$ and $k_{\mathrm{m}}$, it is essential to know the spinodal curve, critical point, correlation length $\xi$, and diffusion coefficient $D$ for this system. The time dependence of $k_{\mathrm{m}}$ may depend on whether the mixture is the critical or off-critical one, as the data on isobutyric acid-water mixtures suggest. ${ }^{2}$ For a quantitative comparison of the data with theories and other experimental data, it is necessary to express the relation between $k_{\mathrm{m}}$ and $t$ in terms of reduced variables scaled by the system- dependent quatities, $\xi$ and $D .^{2-7}$

In the present study, we have measured the osmotic compressibility, correlation length, and diffusion coefficient near but above the cloud point curve of polystyrene-poly(methylphenylsiloxane) mixtures by static and dynamic light scattering, determined the critical concentration and spinodal temperatures, and observed spinodal rings for the critical mixture. The data for the spinodal rings were compared with those of the off-critical mixtures obtained previously. The changes in $k_{\mathrm{m}}$ with $t$ in the critical and off-critical mixtures are discussed on the basis of the scaled form.

\section{EXPERIMENTAL}

\section{Materials}

The samples used here were the same polystyrene (PS) and poly(methylphenylsiloxane) (PMPS) used in previous studies. ${ }^{1}$ The weight-average molecular weight $M_{w}$ and the ratio of $M_{w}$ to the number- 


\section{S. NoJima et al.}

average molecular weight $M_{n}$ were 9,000 and less than 1.06 for PS, and 2,800 and 1.57 for PMPS, respectively.

\section{Light Scattering Measurements above the Cloud Point Curve}

a) Sample Preparation. Desired amounts of PS and PMPS were dissolved in benzene at a total concentration less than $25 \mathrm{wt} \%$, and the solution was filtered into a light-scattering cell through a Millipore filter of $0.20 \mu \mathrm{m}$ nominal pore diameter. The benzene was subsequently allowed to evaporate at about $110^{\circ} \mathrm{C}$ for $50 \mathrm{~h}$. The cloud point of the mixture thus obtained was consistent with that of the sample prepared by mixing the two polymers with no solvent.

b) Apparatus. Light-scattering measurements were carried out by a specially designed spectrometer provided with a photon counting device and an argon-ion laser source (NEC GLG 3200) operating at $488 \mathrm{~nm}$. The single-cripped photoelectroncount autocorrelation function $G^{(2)}(t)$ was measured by a 48 -channel Malvern correlator. The temperature for each measurement was maintained to within $\pm 0.05^{\circ} \mathrm{C}$.

Benzen was used as a reference for computing the Rayleigh ratio $R(\theta)$, assuming the following equation to be valid for vertically polarized incident and scattered light at $t^{\circ} \mathrm{C}$ and $\lambda_{0}$ (vacuum wavelength of light $)=488 \mathrm{~nm}^{7}$

$$
\begin{aligned}
R_{\mathrm{vv}}(90)_{t, \text { Benzene }} & =3.20 \\
\times & 10^{-5}\left[1+0.368 \times 10^{-2}(t-25)\right]
\end{aligned}
$$

Attenuation correction was made for the measured scattered intensity $I_{\text {meas }}$ related to the true scattered intensity $I$ by $I_{\text {meas }}=I \exp (-\tau d)$, where $\tau$ is the tubidity of the solution and $d$ the light path length. $\tau$ was estimated by ${ }^{8}$

$$
\begin{aligned}
\tau= & R(0) \pi\left[\left(2 \alpha^{2}+2 \alpha+1\right)^{-3} \ln (1+2 \alpha)\right. \\
& \left.-2(1+\alpha)^{-2}\right]
\end{aligned}
$$

where $\alpha=2(k \xi)^{2}$ and $k=(4 \pi / \lambda) \sin (\theta / 2)$, with $\xi$ being the correlation length, $\lambda$ the wavelength of light in the solution, and $\theta$ the scattering angle. The maximum attenuation correction was about $20 \%$ for the mixtures studied.

c) Conditions. Static and dynamic light scattering measurements were carried out for mixtures of $23.9,31.4,39.5$ and $50.4 \mathrm{wt} \% \mathrm{PS}$ at several tempera- tures ranging from the cloud point to about $10^{\circ} \mathrm{C}$ above it.

\section{Light Scattering Measurements during the Phase Separation}

The intensity of scattered light during the phase separation was measured for the critical mixture at several temperatures ranging from the critical temperature to $2.5^{\circ} \mathrm{C}$ below it for $20-40 \mathrm{~min}$, using the same apparatus described in the previous paper. ${ }^{1}$ Temperature fluctuation was surpressed to within $\pm 0.05^{\circ} \mathrm{C}$ throughout each measurement.

\section{Methods of Data Analysis}

a) Correlation Length and Spinodal Temperature. The Ornstein-Zernike form ${ }^{9}$

$$
I(0) / I(\theta)=1+\xi^{2} k^{2}
$$

was assumed for estimating the intensity at zero angle $I(0)$ and the correlation length $\xi$ from the angular dependence of intensity $I(\theta)$ in the homogeneous region above the cloud point. The resulting $I(0)$ near the spinodal temperature $T_{\mathrm{s}}$ was expressed by ${ }^{10}$

$$
I(0) \propto\left(\left|T-T_{\mathrm{s}}\right| / T_{\mathrm{s}}\right)^{-\gamma}
$$

with $\gamma=1.26 . T_{\mathrm{s}}$ was determined by extrapolating the plot of $I(0)^{-1 / \gamma} v s$. $T$ to $I(0)^{-1 / \gamma}=0$. The correlation length $\xi$ near $T_{\mathrm{s}}$ was assumed to be expressed by

$$
\xi=\xi_{0}\left(\left|T-T_{\mathrm{s}}\right| / T_{\mathrm{s}}\right)^{-v}
$$

with $v$ and $\xi_{0}$ being the exponent and amplitude, respectively. The values of $v$ and $\xi_{0}$ were obtained by applying the least squares method to the plot of $\log \xi v s . \log \left(\left|T-T_{\mathrm{s}}\right| T_{\mathrm{s}}\right)$, with the spinodal temperature $T_{\mathrm{s}}$ determined above (eq 4 ).

b) Diffusion Coefficient. The normalized correlation function $\left|g^{(1)}(t)\right|$ was calculated from the measured correlation function $G^{(2)}(t)$ by the equation $G^{(2)}(t)=A\left[1+B\left|g^{(1)}(t)\right|^{2}\right]$, where $A$ and $B$ are constants. The data of $\left|g^{(1)}(t)\right|$ were analyzed by the method of cumulants, which gives the average line width $\bar{\Gamma} .{ }^{11}$ The diffusion coefficient $D$ was computed by $D=\bar{\Gamma} / k^{2}$, which, near $T_{\mathrm{s}}$, was assumed to be expressed by

$$
D=D_{0}\left(\left|T-T_{\mathrm{s}}\right| / T_{\mathrm{s}}\right)^{v^{\prime}}
$$

Usually, $D_{0}$ may be regarded as constant. In the present case, however, the temperature dependence 
of $D_{0}$ due to the change in mobility has to be taken into account, since the experimental range of temperature was wide and not removed from the glass transition temperature $T_{\mathrm{g}}$. We assumed that temperature dependence of mobility $M(T)$ could be expressed by the WLF equation ${ }^{12}$

$$
\begin{gathered}
\log D_{0} \propto \log M(T) \\
\propto C_{1}\left(T-T_{\mathrm{g}}\right) /\left[\mathrm{C}_{2}+\left(T-T_{\mathrm{g}}\right)\right]
\end{gathered}
$$

with $C_{1}=17.44$ and $C_{2}=51.6$. The corrected diffusion coefficient $D_{\mathrm{c}}$ defined by

$$
D_{\mathrm{c}}=D M\left(T^{*}\right) / M(T)
$$

was fitted to the power-law relation $D_{\mathrm{c}}=$ $D_{0 \mathrm{c}}\left(\left|T-T_{\mathrm{s}}\right| / T_{\mathrm{s}}\right)^{v^{\prime}}$ by the least squres method, by which we determined the values of the exponent $v^{\prime}$ and corrected amplitude $D_{0 \mathrm{c}}$

$$
D_{0 \mathrm{c}}=D_{0} M\left(T^{*}\right) / M(T)
$$

where the reference temperature $T^{*}$ was chosen to be $T_{\mathrm{s}}$.

\section{RESULTS AND DISCUSSION}

\section{Spinodal Temperatures and the Critical Point}

Figure 1 shows the plot of $I(0)^{-1 / \gamma} v s$. $T$ for the $23.9 \mathrm{wt} \% \mathrm{PS}$ mixture. The spinodal temperature $T_{\mathrm{s}}$ could be estimated from such plots for the mixtures of $23.9,31.4$, and $39.5 \mathrm{wt} \% \mathrm{PS}$ except for the mixture of $50.4 \mathrm{wt} \% \mathrm{PS}$ for which the spinodal temperature was more than $10^{\circ} \mathrm{C}$ lower than the cloud point temperature. The spinodal temperatures thus obtained are shown in Figure 2, along with the cloud point curve and glass transition points measured previously. ${ }^{13}$ The critical concentration was about $31 \mathrm{wt} \% \mathrm{PS}$ at which the spinodal and cloud point curves touched tangentially. In the vicinity of the critical point, the cloud point curve is slightly concave, possibly as a result of the rather broad distribution of molecular weight of the PMPS sample. $^{14,15}$

\section{Correlation Length and Diffusion Coefficient}

The plots of $\log \xi$ and $\log D_{\mathrm{c}}$ against $\log \left(T-T_{\mathrm{s}}\right)$ were almost linear, as can be seen from Figures 3 and 4 for the $23.9 \mathrm{wt} \% \mathrm{PS}$ mixture. The values of $\xi_{0}$, $v, D_{0 c}$, and $v^{\prime}$ obtained by the linear least-squares method are listed in Table I.

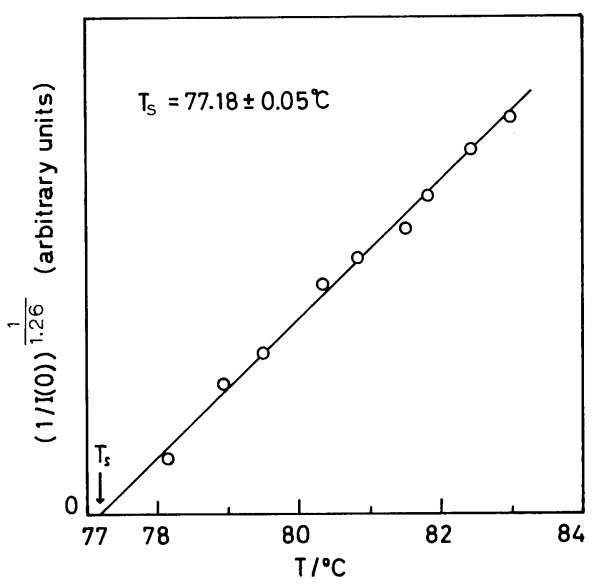

Figure 1. Plots of $I(0)^{-1 / \gamma}$ against $T$ for the mixture of $23.9 \mathrm{wt} \%$ PS.

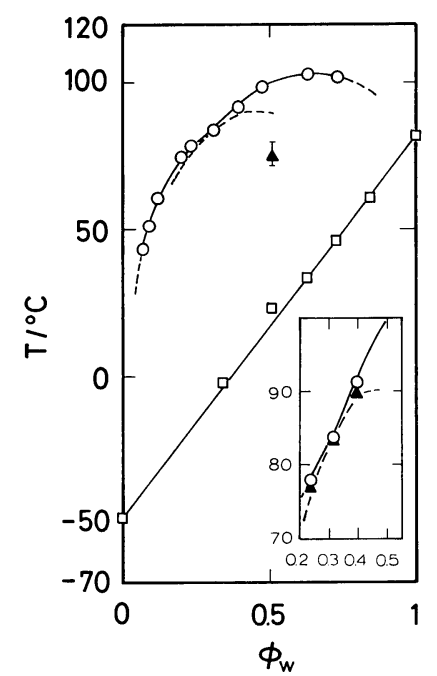

Figure 2. Cloud points $(\bigcirc)$, spinodal points $(\boldsymbol{\Delta})$, and glass transition points ( $\square$ ) of PS and PMPS mixtures.

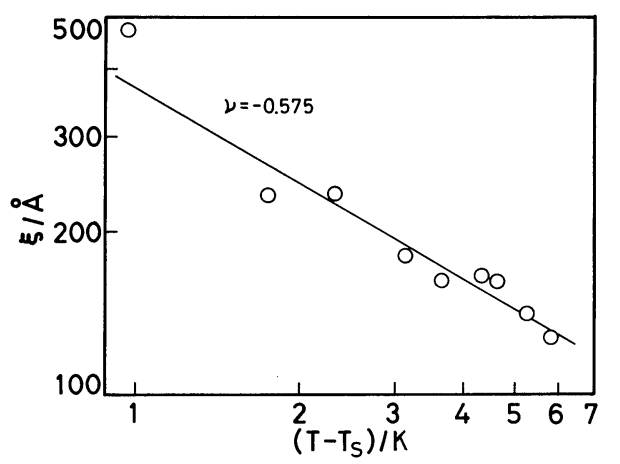

Figure 3. Plots of $\log \xi$ and $\log \left(T-T_{\mathrm{s}}\right)$ for the mixture of $23.9 \mathrm{wt} \% \mathrm{PS}$. 


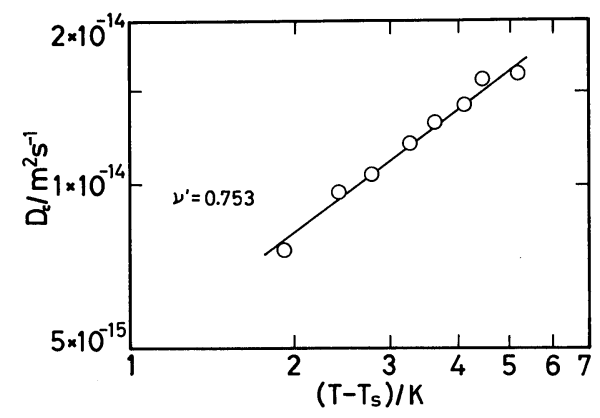

Figure 4. Plots of $\log D_{\mathrm{c}} v s \cdot \log \left(T-T_{\mathrm{s}}\right)$ for the mixture of $23.9 \mathrm{wt} \% \mathrm{PS}$.

Table I. Parameters in power-laws for the correlation length $\xi$ and the diffusion coefficient $D$ (eq 5 and 6 with eq $8^{\prime}$ )

\begin{tabular}{|c|c|c|c|c|}
\hline$C$ & $\xi_{0}$ & & $D_{0 \mathrm{C}}$ & \\
\hline $\mathrm{wt} \% \mathrm{PS}$ & $\AA$ & & $\mathrm{m}^{2} \mathrm{~s}^{-1}$ & \\
\hline 23.9 & 12.7 & 0.58 & $4.44 \times 10^{-13}$ & 0.75 \\
\hline 31.4 & 10.7 & 0.58 & 0.628 & 0.37 \\
\hline 39.5 & 5.1 & 0.75 & 0.594 & 0.45 \\
\hline
\end{tabular}

Phase Separation Behavior of Critical and OffCritical Mixtures

The time dependence of the maximum intensity $I_{\mathrm{m}}$ and the corresponding wave number $k_{\mathrm{m}}$ for the critical mixture are shown in Figures 5 and 6, respectively. The time evolution of $I_{\mathrm{m}}$ and $k_{\mathrm{m}}$ can be represented by the power-law, $I_{\mathrm{m}} \propto t^{\theta}$ and $k_{\mathrm{m}} \propto t^{\phi}$, as was the case for off-critical mixtures. ${ }^{1}$ Table II summarizes the values of $\theta$ and $\phi$ for the critical mixture at the various quench depths $\Delta T$ studied, where $\Delta T$ is the extent to which the temperature differs with the cloud point. It can be seen that $\theta=$ $2.66( \pm 0.43)$ and $\phi=-0.90( \pm 0.20)$ at any $\Delta T$, while $\theta=0.69( \pm 0.17)$ and $\phi=-0.29( \pm 0.05)$ for off-critical mixtures at any $\Delta T$ and concentration. ${ }^{1}$ The values of $\theta$ and $\phi$ for critical mixture are approximately three times as large as those for the off-critical mixtures. This difference in the values of $\theta$ and $\phi$ for the critical and off-critical mixtures was also found by Wong and Knobler ${ }^{2}$ in binary mixtures of isobutyric acid and water.

Our previous phase-separation experiments on off-critical mixtures dealt with the metastable region down to the unstable region enclosed by the spino-

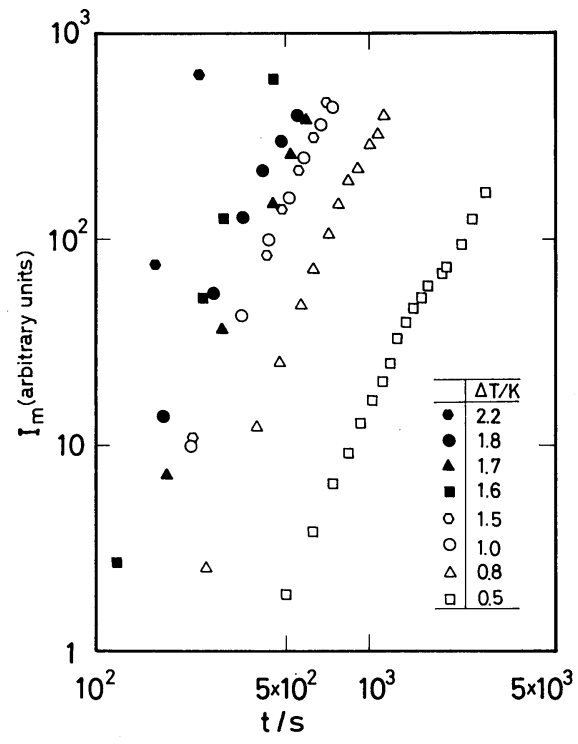

Figure 5. Maximum intensity $I_{\mathrm{m}}$ as a function of $t$ for the mixture of $31.4 \mathrm{wt} \% \mathrm{PS}$ (critical mixture).

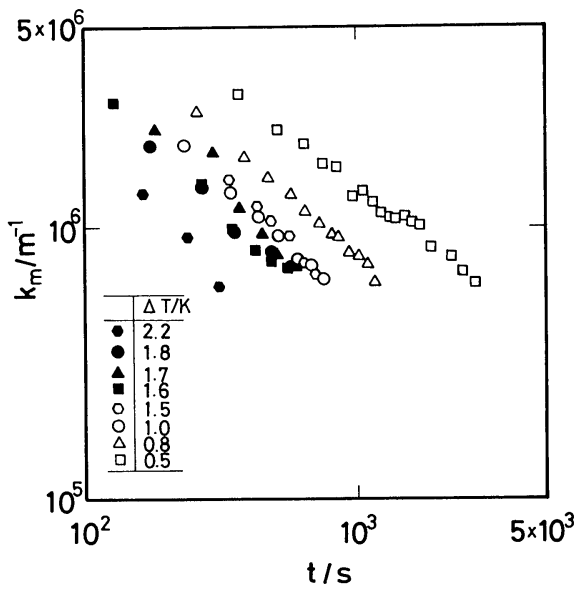

Figure 6. The wave number for $I_{\mathrm{m}}, k_{\mathrm{m}}$, as a function of $t$ for the mixture of $31.4 \mathrm{wt} \% \mathrm{PS}$.

dal curve. However, no particular difference in the phase separation behavior was found between these two regions, except at small $\Delta T$ for 50.4 and 55.0 $\mathrm{wt} \% \mathrm{PS}$ mixtures.

This finding seems to support the theory of Binder et al., ${ }^{16}$ which predicts phase separation behavior characterized by smooth change in going from the metastable region to the unstable region. In the Cahn theory, ${ }^{17}$ the spinodal temperature is a singular point at which the wavelength of con- 
Table II. Exponents in power-laws for $I_{\mathrm{m}}$ and $k_{\mathrm{m}}$ $\left(I_{\mathrm{m}} \propto t^{\theta}, k_{\mathrm{m}} \propto t^{\phi}\right)$ of the critical mixture

\begin{tabular}{|c|c|c|c|}
\hline$C$ & $\Delta T$ & & \\
\hline $\mathrm{wt} \% \mathrm{PS}$ & $\mathrm{K}$ & & \\
\hline \multirow{8}{*}{31.4} & 2.2 & 3.79 & -1.24 \\
\hline & 1.8 & 2.26 & -0.97 \\
\hline & 1.7 & 2.60 & -1.01 \\
\hline & 1.6 & 3.03 & -0.98 \\
\hline & 1.5 & 2.49 & -1.01 \\
\hline & 1.0 & 2.49 & -0.76 \\
\hline & 0.8 & 2.51 & -0.92 \\
\hline & 0.5 & 2.10 & -0.77 \\
\hline Average & & 2.66 & -0.96 \\
\hline
\end{tabular}

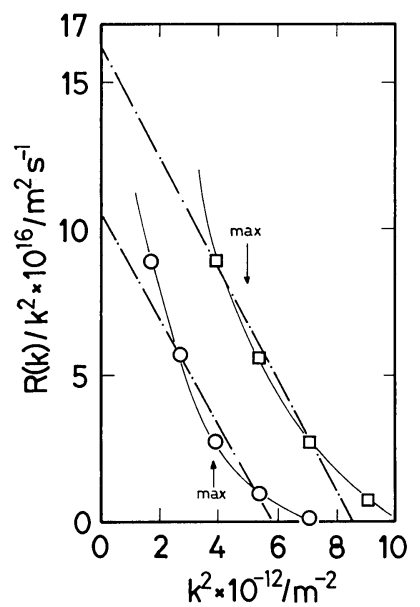

Figure 7. The values of $R(k) / k^{2}$ as a function of $k^{2}$ at selected values of $t: \square, 120 \mathrm{~s}$ after quench; $\bigcirc, 268 \mathrm{~s}$ after quench. centration fluctuations becomes infinite.

Though not in conformity with the Cahn theory ${ }^{17}$ because of the dependence of $k_{\mathrm{m}}$ on $t,{ }^{1}$ the present data for short periods of time following quenching may permit an approximate evaluation of the diffusion coefficient $D$ following the previous procedure. ${ }^{1}$ The value of $D$ thus obtained may be compared with that calculated from dynamic light scattering measurements on the assumption that eq 6 with eq 7-8 $8^{\prime}$ holds in the two-phase region $\left(T-T_{\mathrm{s}}<0\right)$. Figure 7 shows the plot of $R(k) / k^{2} v s$. $k^{2}$ for the mixture of $23.9 \mathrm{wt} \% \mathrm{PS}$ at $\Delta T=5.4^{\circ} \mathrm{C}$, where $R(k)$ is the amplification factor (see ref 1 or 17). The intercepts of the dot-dash lines on the ordinate axis give $-1.0 \sim-1.7 \times 10^{-15} \mathrm{~m}^{2} \mathrm{~s}^{-1}$ for $D$, while the dynamic light scattering measurements yield $D=-9.6 \times 10^{-15} \mathrm{~m}^{2} \mathrm{~s}^{-1}$.

The former $D$ from the Cahn theory is much smaller than the latter from dynamic light scattering. One possible explanation for this discrepancy is that the experimental range of separation time may greatly exceed that prescribed by the theory. The absolute value of $D$ estimated by the theory is expected to become larger in observing the earlier stage.

\section{Scaling Analysis of Phase Separation Behavior}

The wave number $k_{\mathrm{m}}$ at the maximum intensity of scattered light and the separation time $t$ can be scaled to dimension-less variables $q_{\mathrm{m}}$ and $\tau$ :

$$
q_{\mathrm{m}}=k_{\mathrm{m}} \xi
$$

and

$$
\tau=t /\left(\xi^{2} / D\right)
$$

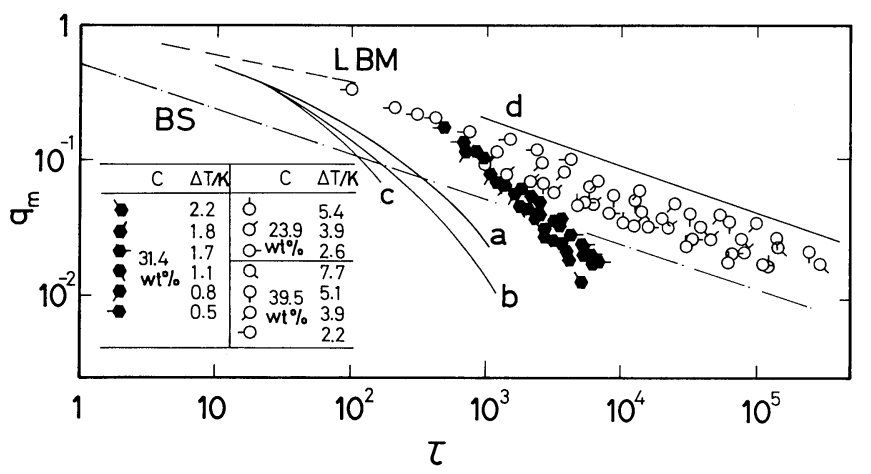

Figure 8. Plots of $q_{\mathrm{m}} v s . \tau$ for $23.9,31.4$, and $39.5 \mathrm{wt} \% \mathrm{PS}$ mixtures:---, the LBM theory; -----, the BS theory; a, the critical mixture of $\mathrm{L}-\mathrm{W}^{2}$; b, the critical mixture of $\mathrm{I}-\mathrm{W}^{5}$; $\mathrm{c}$, the critical mixture of PDMS$\mathrm{DC}^{6} ; \mathrm{d}$, the off-critical mixture of $\mathrm{I}-\mathrm{W} .^{5}$ 


\section{S. Nojima et al.}

where $\xi$ and $D$ are the correlation length and diffusion coefficient in the one-phase region. ${ }^{2,5}$ The values of $\xi$ and $D$ for the scaling at a given temperature $T\left(<T_{\mathrm{s}}\right)$ were calculated from eq 5-8 with the parameters listed in Table I. Figure 8 shows the log$\log$ plots of $q_{\mathrm{m}} v s . \tau$ for the mixtures of 23.9, 31.4, and $39.5 \mathrm{wt} \% \mathrm{PS}$, but some of the data at small $\Delta T$ have not been included. The data points at various $\Delta T$ and concentrations form two composite lines: one for the critical mixture with a slope of almost unity in the time range $10^{3}<\tau<10^{4}$, and the other for off-critical mixtures with a slope of about $1 / 3$ in $10^{2}<\tau<10^{5}$.

The broken and dot-dash lines in Figure 8 represent the predictions of a non-linear theory of Langer, Bar-on, and Miller (LBM theory) ${ }^{18}$ and a cluster dynamic theory of Binder and Stauffer (BS theory), ${ }^{19}$ respectively. The LBM theory is limited to the time range $4<\tau<100$, which is beyond the scope of the present experiment. The BS theory is applicable to the whole range of separation time, and describes quite well our experimental results for off-critical mixtures.

The solid curves $\mathrm{a}, \mathrm{b}$, and $\mathrm{c}$ show the experimental results for the critical mixtures of 2-6 lutidinewater $(\mathrm{L}-\mathrm{W}),{ }^{5}$ isobutyric acid-water $(\mathrm{I}-\mathrm{W}),{ }^{2}$ and polydimethylsiloxane-diethylcarbonate (PDMSDC), ${ }^{6}$ respectively. All these curves indicate rapid decrease in $q_{\mathrm{m}}$ at larger $\tau$. The present data for the critical mixture and these curves have approximately the same slopes in a comparable range of $q_{\mathrm{m}}$, but do not overlap with at large $\tau$.

The values of $q_{\mathrm{m}}$ for the off-critical mixtures of I$\mathrm{W}^{3}$ decrease with a slope of about $1 / 3$ in the time range $10^{3}<\tau<10^{7}$ as indicated by the solid line $\mathrm{d}$. This is consistent with the present data for offcritical mixtures.

The features of separation behavior of the present critical and off-critical mixtures are quite similar to those of small molecules. This seems reasonable since the wavelength of predominant concentration fluctuation is much larger than the size of polymers in the present case. When the inverse is the case, the phase separation mechanism for polymers should be different from that for small molecules. ${ }^{20,21}$ It should be noted, however, that, in the present study, the observation times, the quench depths, and concentration differences from the critical point were much larger than those in the experiments on systems of small molecules (and polymer solutions), in which phase separation process could be observed experimentally only near critical points.

\section{REFERENCES}

1. S. Nojima, K. Tsutsumi, and T. Nose, Polym. J., 14, 225 (1982).

2. N. C. Wong and C. M. Knobler, J. Chem. Phys., 66, 4707 (1977); ibid., 69, 725 (1978).

3. A. J. Schwartz, J. S. Huang, and W. I. Goldburg, $J$. Chem. Phys., 62, 1847 (1975).

4. W. I. Goldburg, C. H. Shaw, J. S. Huang, and M. S. Pilant, J. Chem. Phys., 68, 484 (1978).

5. Y. C. Chou and W. I. Goldburg, Phys. Rev., A20, 2105 (1979).

6. N. Kuwahara, M. Tachikawa, K. Hamano, and Y. Kenmochi, Phys. Rev., A25, 3449 (1982).

7. T. Nose and B. Chu, Macromolecules, 12, 590 (1979).

8. V. G. Puglielle and N. C. Ford, Jr., Phys. Rev. Lett., 25, 143 (1970).

9. L. S. Ornstein and F. Zernike, Proc. Acad. Sci., Amsterdam, 17, 793 (1914).

10. N. Kuwahara, D. V. Fenby, M. Tamsky, and B. Chu, J. Chem. Phys., 55, 1140 (1971).

11. D. E. Koppel, J. Chem. Phys., 57, 4814 (1972).

12. M. L. Williams, R. F. Landel, and J. D. Ferry, J. Am. Chem. Soc., 77, 3701 (1955); J. D. Ferry, "Viscoelastic Properties of Polymers," 2nd Ed., John Wiley and Sons, New York, N. Y., 1970.

13. S. Nojima and T. Nose, Polym. J., 14, 269 (1982).

14. R. Koningsveld and L. A. Kleintjens, J. Polym. Sci., Polym. Symp., No. 61, 221 (1977).

15. R. Koningsveld and L. A. Kleintjens, Br. Polym. J., 9, 212 (1977).

16. K. Binder, C. Billotet, and P. Mirold, Z. Physik, B30, 183 (1978).

17. J. W. Cahn, J. Chem. Phys., 42, 93 (1965).

18. J. S. Langer, M. Bar-on, and H. D. Miller, Phys. Rev., A11, 1417 (1975).

19. K. Binder and D. Stauffer, Adv. in Physics, 25, 343 (1976).

20. P. G. de Gennes, J. Chem. Phys., 72, 4756 (1980).

21. P. Pincus, J. Chem. Phys., 75, 1996 (1981). 